\title{
Elaidic, vaccenic, and rumenic acid status during pregnancy: association with maternal plasmatic LC-PUFAs and atopic manifestations in infants
}

\author{
Aida Maribel Chisaguano ${ }^{1,2}$, Rosa Montes ${ }^{1,2}$, Ana Isabel Castellote ${ }^{1,2}$, Eva Morales ${ }^{3,4,5,6}$, Jordi Júlvez ${ }^{3,4,5,6}$, Jesús Vioque , \\ Jordi Sunyer 3,4,5,6,8 and Maria Carmen López-Sabater ${ }^{1,2}$
}

BACKGROUND: Few studies have explored whether fetal exposure to trans fatty acids (TFAs) influences the inception of atopic diseases. The aim of this study was to investigate the relationship between the concentration of specific TFAs (elaidic, vaccenic, and rumenic acids) in maternal plasma and the risk of developing atopic manifestations in the first year of life.

METHODS: A subsample from a population-based pregnancy cohort of the INMA Project was analyzed. Maternal intake of fatty acids was assessed by a food-frequency questionnaire (75.5\% of the cohort). TFAs and $n-3$ and $n-6$ long-chain polyunsaturated fatty acids were measured in samples of plasmatic phospholipids at $12 \mathrm{wk}$ of pregnancy. Information regarding eczema and wheeze in offspring was obtained through questionnaires at ages 6 and 14 mo.

RESULTS: Elaidic acid correlated negatively with n-3 longchain polyunsaturated fatty acids (total, eicosapentaenoic acid, and docosahexaenoic acid), and rumenic acid positively with both n-3 and n-6 long-chain polyunsaturated fatty acids in maternal plasma. Neither of these two fatty acids was associated with the risk of atopic eczema or wheeze in offspring in the first year of life. However, a higher vaccenic acid level was found to be linked to a lower risk of atopic eczema.

CONCLUSION: High vaccenic acid concentrations in maternal plasma may protect offspring against atopic eczema in infancy.

$\mathbf{T}$ he prevalence of atopic diseases (atopic eczema, allergic rhinoconjunctivitis, and asthma) in young children has increased rapidly in recent decades. Currently, a third of children in Western countries have confirmed symptoms of these conditions (1). Epidemiological and clinical studies indicate that changes in fatty acid (FA) intake by expectant mothers may contribute to the development of atopic disorders during infancy and early childhood. Prenatal exposure to n-6 and n-3 essential fatty acids (linoleic acid (LA, C18:2 n-6), a-linolenic acid (ALA, C18:3 n-3)) and long-chain polyunsaturated fatty acids (LC-PUFAs) has been studied extensively. It has been shown that n-3 LC-PUFAs, such as eicosapentaenoic acid (EPA, C20:5 n-3) and docosahexaenoic acid (DHA, C20:6 n-3) exert protective effects against atopic eczema, while the opposite is true for n-6 LC-PUFAs, such as arachidonic acid (AA, C20:4 n-6) (2-7). Nevertheless, little attention has been devoted to evaluating the effects of maternal intake of trans fatty acids (TFAs) on the prevalence of atopic diseases in early infancy. It has been proposed that TFAs contribute to allergic response by altering lipid metabolism and the phospholipid composition of cell membranes. These effects alter membrane permeability, eicosanoid production (by modifications of substrate levels, such as AA and DHA, which are substrates in the production of proinflammatory and anti-inflammatory eicosanoids, respectively), and gene expression (8-10).

TFAs include unsaturated and polyunsaturated fatty acids with at least one double bond in the trans configuration. Dietary TFAs come from two sources: those derived from industrial production by partial hydrogenation of vegetable oils (e.g., elaidic acid, t9-C18:1) and those from natural sources, such as ruminant-derived foods (e.g., vaccenic acid (t11-C18:1) and rumenic acid (c9t11-CLA)) (11). Clinical evidence indicates that natural TFAs have distinct properties from those of synthetically produced partially hydrogenated vegetable oils. The inclusion of vaccenic and rumenic acids in the diet has beneficial effects for conditions such as cardiovascular disease, metabolic syndrome, and cancer $(12,13)$.

Dairy fat is rich in vaccenic and rumenic acids. Convergent lines of research on dairy product consumption have indirectly evaluated the link between ruminant TFAs and atopic diseases. As an example, dairy products have been found to show a good correlation with blood concentrations of rumenic

'Department of Nutrition and Food Science, Faculty of Pharmacy, University of Barcelona, Barcelona, Spain; ${ }^{2}$ CIBER Fisiopatología de la Obesidad y Nutrición (CIBERobn), Instituto de Salud Carlos III (ISCIII), Spanish Government, Madrid, Spain; ${ }^{3}$ Center for Research in Environmental Epidemiology (CREAL), Barcelona, Spain; ${ }^{4}$ Hospital del Mar Research Institute (IMIM), Barcelona, Spain; ${ }^{5}$ CIBER Epidemiología y Salud Pública (CIBERESP), Instituto de Salud Carlos III (ISCIII), Spanish Government, Madrid, Spain; ${ }^{6}$ Universitat Pompeu Fabra (UPF), Barcelona, Spain; ${ }^{7}$ Departamento de Salud Pública, Universidad Miguel Hernández, Alicante, Spain; ${ }^{8}$ Center for Genomic Regulation (CRG), Barcelona, Spain. Correspondence: Maria Carmen López-Sabater (mclopez@ub.edu) 
acid, thus making these concentrations an appropriate marker of rumenic acid intake (14). Studies addressing the relationship between TFA consumption in pregnant women and the prevalence of atopic diseases in newborns remain controversial. On the one hand, the consumption of organic dairy products has been associated with a lower risk of eczema in a Dutch cohort (15). Moreover, a study in a Danish cohort identified a link between the intake of low-fat yoghurt and an increased risk of asthma and allergic rhinitis, while whole milk appeared protective for early-life outcomes (16). Other studies have revealed that a high intake of dairy products by expectant mothers may decrease the risk of wheeze in the first year of life (17). On the other hand, high maternal intake of butter and butter spreads during pregnancy were associated with an increased risk of allergic rhinitis in offspring at $5 \mathrm{y}$ of age (3). The latter observation is interesting given that previous studies in an Italian cohort did not find an association between the maternal intake of butter or margarine and atopy in offspring (18).

Animal models have been used to evaluate the real influence of the consumption of dairy products, such as fat-enriched milk, on atopy. Those studies revealed that the two major TFAs, vaccenic acid and rumenic acid, attenuate allergic dermatitis and suppress airway inflammation $(19,20)$; however, no data about specific TFAs have been reported for humans yet.

To better understand the contribution of individual TFAs during pregnancy to the development of atopic disease in infants, here we studied whether relative concentrations of elaidic, vaccenic, and rumenic FAs in maternal plasma are associated with risk of eczema and wheeze in offspring during the first year of life.

\section{RESULTS}

\section{Study Population Characteristics}

The distribution of baseline characteristics of mother-infant pairs participating in the INMA-Sabadell cohort study is given in Table 1. A total of 496 women (75.5\%) answered the questions about dietary intake. Most women had an average educational level (42.5\%), and the mean maternal age at birth was 31.4 y. Of the participants, $14.8 \%$ reported smoking during pregnancy. The prevalence of maternal allergy and atopic manifestations was 30.2 and $38.5 \%$, respectively. Of the babies born to the participants, $49.1 \%$ were males, and the predominant breastfeeding period lasted between 16 and $24 \mathrm{wk}$. The prevalence of atopic eczema and wheezing in the first year of life was 20.8 and $35.8 \%$, respectively.

For the determination of maternal plasma phospholipids composition, a subset of maternal plasma $(n=274)$ was used. This subsample was representative since no statistically significant differences were found with general population for any of the studied characteristics (data not shown).

\section{Maternal Fatty Acid Intake}

The food-frequency questionnaire (FFQ) was developed to estimate the intake of fat. Using this information, we determined the FAs most consumed. The intake of the FAs is expressed in
Table1. Characteristics of the INMA-Sabadell cohort study

Characteristics

\begin{tabular}{|c|c|}
\hline \multicolumn{2}{|l|}{ Maternal characteristics } \\
\hline Allergy $(\lg E>0.35 \mathrm{Uk} / \mathrm{l})^{\mathrm{a}}$, yes (\%) & 30.2 \\
\hline Maternal history of atopic manifestations ${ }^{b}$, yes (\%) & 38.5 \\
\hline \multicolumn{2}{|l|}{ Education (\%) } \\
\hline Low (primary or less) & 28.2 \\
\hline Medium (secondary) & 42.5 \\
\hline High (university or more) & 29.4 \\
\hline Smoking in pregnancy, yes (\%) & 14.8 \\
\hline Age at delivery (mean \pm SD) & $31.4(4.37)$ \\
\hline \multicolumn{2}{|l|}{ Children characteristics } \\
\hline Sex, male (\%) & 49.1 \\
\hline \multicolumn{2}{|l|}{ Predominant breastfeeding, weeks (\%) } \\
\hline 0 & 21.1 \\
\hline$>0-16$ & 29.8 \\
\hline $16-24$ & 40.4 \\
\hline$>24$ & 8.7 \\
\hline Atopic eczema (\%) & 20.8 \\
\hline Wheeze (\%) & 35.8 \\
\hline \multicolumn{2}{|l|}{ Fatty acid intake $(g / d)(\text { mean } \pm S D)^{c}$} \\
\hline $\mathrm{C} 16: 0$ & $16.00 \pm 2.53$ \\
\hline C18:0 & $6.38 \pm 1.28$ \\
\hline C18:1 n-9 & $42.14 \pm 8.35$ \\
\hline C18:2 n-6 (LA) & $11.54 \pm 2.60$ \\
\hline C18:3 n-3 (ALA) & $1.10 \pm 0.19$ \\
\hline$C 20: 4 n-6(A A)$ & $0.16 \pm 0.05$ \\
\hline$C 20: 5 n-3$ (EPA) & $0.15 \pm 0.09$ \\
\hline $\mathrm{C} 22: 6 \mathrm{n}-3(\mathrm{DHA})$ & $0.27 \pm 0.17$ \\
\hline$\sum$ Trans & $1.32 \pm 0.58$ \\
\hline$\Sigma n-3$ & $1.52 \pm 0.33$ \\
\hline$\Sigma \mathrm{n}-6$ & $11.70 \pm 2.61$ \\
\hline
\end{tabular}

AA, arachidonic acid; ALA, a-linolenic acid; DHA, docosahexaenoic acid; EPA, eicosapentaenoic acid; IgE, immunoglobulin E; LA, linoleic acid.

algE antibodies against mite, cat dander, and Poaceae plants (one or more positive

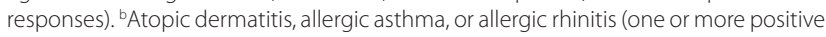
responses). cNutrient intakes were naturally log transformed ( $n=496)$.

gram per day (Table 1). Oleic acid (C18:1 n-9, $42.1 \mathrm{~g} / \mathrm{d})$ was the most abundant FA in the diet of pregnant women, followed by the saturated FAs palmitic (C16:0, $16.0 \mathrm{~g} / \mathrm{d})$ and stearic (C18:0, $6.38 \mathrm{~g} / \mathrm{d})$ acids. The predominant $\mathrm{n}-6 \mathrm{FA}$ in the diet was LA $(11.54 \mathrm{~g} / \mathrm{d})$, followed by AA $(0.16 \mathrm{~g} / \mathrm{d})$. Within the $\mathrm{n}-3$ series, the main FA was ALA $(1.10 \mathrm{~g} / \mathrm{d})$, followed by DHA $(0.27 \mathrm{~g} / \mathrm{d})$ and EPA $(0.15 \mathrm{~g} / \mathrm{d})$. The dietary intake of total TFAs and total $n-3$ and total n-6 PUFAs was $1.32,1.52$, and $11.72 \mathrm{~g} / \mathrm{d}$, respectively.

\section{Relationship Between Maternal Fatty Acid Intake and Plasma Fatty Acid Status}

The predominant n-6 FA in maternal plasma was LA (22.94\%), while the dominant n-3 FA was DHA (4.56\%). The main 
Table 2. Fatty acid concentrations in maternal plasma phospholipids

\begin{tabular}{|c|c|}
\hline Fatty acid & Maternal plasma $($ mean \pm SD) \\
\hline C16:0 & $30.34 \pm 1.80$ \\
\hline C18:0 & $12.11 \pm 1.23$ \\
\hline C18:1 n-9 & $9.20 \pm 1.61$ \\
\hline C18:2n-6 (LA) & $22.94 \pm 3.41$ \\
\hline C18:3 n-6 (GLA) & $0.05 \pm 0.03$ \\
\hline$C 20: 2 n-6(E D A)$ & $0.46 \pm 0.10$ \\
\hline C20:3 n-6 (DGLA) & $3.50 \pm 0.87$ \\
\hline$C 20: 4 n-6(A A)$ & $11.36 \pm 1.96$ \\
\hline C22:4 n-6 (DTA) & $0.38 \pm 0.14$ \\
\hline C22:5 n-6 (DPA) & $0.40 \pm 0.17$ \\
\hline C18:3 n-3 (ALA) & $0.12 \pm 0.04$ \\
\hline C20:5 n-3 (EPA) & $0.41 \pm 0.33$ \\
\hline C22:5 n-3 (DPA) & $0.47 \pm 0.12$ \\
\hline $\mathrm{C} 22: 6 \mathrm{n}-3(\mathrm{DHA})$ & $4.56 \pm 0.90$ \\
\hline C18:1 t9 & $0.14 \pm 0.05$ \\
\hline C18:1 t11 & $0.18 \pm 0.06$ \\
\hline CLA c9t11 & $0.08 \pm 0.03$ \\
\hline$\sum \operatorname{Trans}^{\mathrm{b}}$ & $0.80 \pm 0.20$ \\
\hline$\Sigma n-3^{c}$ & $5.55 \pm 1.16$ \\
\hline LC-PUFA n-3 & $5.43 \pm 1.16$ \\
\hline$\Sigma n-6^{e}$ & $39.10 \pm 2.35$ \\
\hline LC-PUFA n- $6^{f}$ & $16.10 \pm 2.32$ \\
\hline $\mathrm{SFA}^{\mathrm{g}}$ & $43.14 \pm 1.44$ \\
\hline $\mathrm{MFA}^{\mathrm{h}}$ & $11.11 \pm 1.73$ \\
\hline PUFA $^{i}$ & $44.87 \pm 1.92$ \\
\hline
\end{tabular}

$\mathrm{AA}$, arachidonic acid; ALA, a-linolenic acid; DGLA, dihomo-gamma-linolenic acid; DHA, docosahexaenoic acid; DPA, docosapentaenoic acid; DTA, docosatetraenoic acid; EDA, eicosadienoic acid; EPA, eicosapentaenoic acid; GLA, gamma linolenic acid; LA, linoleic acid; LC-PUFA, long-chain polyunsaturated fatty acid.

aMaternal plasma $(n=274) .{ }^{b} \sum \operatorname{Trans}\left(\sum \mathrm{C} 16: 1 \mathrm{t}+\sum \mathrm{C} 18: 1 \mathrm{t}+\sum \mathrm{C} 18: 2 \mathrm{t}\right)$.

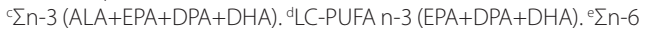

(LA+GLA+EDA+DGLA+AA+DTA+DPA). fLC-PUFA n-6 (EDA+DGLA+AA+DTA+DPA).

${ }^{2}$ SFA (C14:0+C16+C17:0+C18:0+C20:0). ${ }^{n} \mathrm{MFA}(\mathrm{C} 16: 1 \mathrm{n}-9+\mathrm{C} 16: 1 \mathrm{n}-7+\mathrm{C} 18: 1 \mathrm{n}-9+\mathrm{C} 18: 1$ n-7+C20:1 n-9). IPUFA (C20:3n-9+ALA+EPA+DPA+DHA+LA+GLA+EDA+DGLA+AA+D TA+DPA).

saturated FA was palmitic acid (C16:0, 30.34\%) followed by stearic acid (C18:0, 12.11\%). Higher plasma concentrations of vaccenic (C18:1 $t 11,0.18 \%)$ acid were found relative to elaidic (C18:1 t9, 0.14\%) and rumenic (C18.2 c9t11, 0.08\%) acids (Table 2).

Pearson's correlation coefficients between FA intake estimated by the FFQ and maternal FA in plasma phospholipids showed a positive correlation between the intake of stearic acid, LA, EPA, and DHA during first trimester of pregnancy and the concentrations of these FAs in maternal plasma $(P<$ 0.01 ; Table 3$)$. The same behavior was observed for the sum of total n-3 and n-6 FAs $(P<0.01)$.

No association was found between maternal intake of total TFAs and the concentrations of total TFAs $(r=-0.010$; $P=0.865)$, elaidic acid $(r=104 ; P=0.086)$, or vaccenic acid $(r=-0.065 ; P=0.286)$ in maternal plasma. However, a negative correlation between maternal intake of total TFAs and
Table 3. Pearson correlations between plasma fatty acid concentrations and dietary intake in pregnant women of the INMASabadell cohort study

\begin{tabular}{|c|c|c|}
\hline Fatty acid (diet) & $r$ & $P$ value \\
\hline C16:0 & -0.168 & $* *$ \\
\hline C18:0 & 0.150 & * \\
\hline C18:1 n-9 & 0.115 & 0.058 \\
\hline C18:2 n-6 (LA) & 0.150 & $*$ \\
\hline C18:3 n-3 (ALA) & 0.046 & 0.451 \\
\hline C20:4 n-6 (AA) & -0.089 & 0.142 \\
\hline C20:5 n-3 (EPA) & 0.334 & $* *$ \\
\hline C22:6 n-3 (DHA) & 0.279 & $* *$ \\
\hline$\Sigma$ Trans & -0.010 & 0.865 \\
\hline$\sum n-3$ & 0.190 & $* *$ \\
\hline$\sum n-6$ & 0.133 & * \\
\hline \multicolumn{3}{|l|}{$\sum$ Trans } \\
\hline C18:1 t9 & 0.104 & 0.086 \\
\hline C18:1 t11 & -0.065 & 0.286 \\
\hline CLA c9t11 & -0.160 & $* *$ \\
\hline \multicolumn{3}{|c|}{$\begin{array}{l}\text { AA, arachidonic acid; ALA, } a \text {-linolenic acid; DHA, docosahexaenoic acid; EPA, } \\
\text { eicosapentaenoic acid; LA, linoleic acid. } \\
\text { Associations are presented as Pearson's rank correlation coefficients }\left({ }^{*} P<0.05 ;{ }^{* *} P<\right. \\
0.01 \text {. }\end{array}$} \\
\hline
\end{tabular}

rumenic acid concentration in maternal plasma was observed $(r=-0.160 ; P<0.01)$.

\section{Relationship Between TFAs and Long-Chain Polyunsaturated Fatty Acids in Maternal Plasma}

Negative correlations were found between elaidic acid and n-3 LC-PUFAs (total, EPA, and DHA; $P<0.01$ ). Nevertheless, rumenic acid was positively correlated with EPA, DPA, and n-3 LC-PUFAs. No correlations between vaccenic acid and either n-6 or n-3 LC-PUFAs were found (Table 4). Within TFAs, rumenic and vaccenic acid were positively associated in maternal plasma $(r=0.488 ; P<0.01)$ (data not shown).

\section{Maternal Plasma TFAs and Infant Atopic Outcomes}

Risk of atopic eczema in 1-y-old children decreased with increased vaccenic acid in maternal plasma (Table 5). The lowest risk was observed in the highest tertile (odds ratio = 0.373; IC-95 (0.171-0.818); $P$ for trend $=0.035)$. We found no association between elaidic acid (odds ratio $=0.841$; IC-95 $(0.396-1.788)$; $P$ for trend $=0.901)$ and rumenic acid (odds ratio $=0.963$; IC-95 $(0.456-2.032) ; P$ for trend $=0.981)$ and atopic eczema in early life.

Furthermore, wheeze in the first year of life was not associated with maternal levels of elaidic, vaccenic, or rumenic acids during pregnancy (Table 6).

\section{DISCUSSION}

During pregnancy, the transport of TFAs across the placenta is determined by maternal dietary intake (21). First, we evaluated the FA intake of pregnant women during the first trimester of 
Table 4. Pearson correlations between trans fatty acids and LC-PUFAs in maternal plasma phospholipids

\begin{tabular}{|c|c|c|c|c|c|c|c|c|c|}
\hline Fatty acid & $\begin{array}{l}\text { C20:3 n-6 } \\
\text { (DGLA) }\end{array}$ & $\begin{array}{c}C 20: 4 \\
n-6(A A)\end{array}$ & $\begin{array}{l}\text { C22:4 n-6 } \\
\text { (DTA) }\end{array}$ & $\begin{array}{l}\text { C22:5 n-6 } \\
\text { (DPA) }\end{array}$ & $\begin{array}{l}C 20: 5 n-3 \\
\text { (EPA) }\end{array}$ & $\begin{array}{l}\text { C22:5 n-3 } \\
\text { (DPA) }\end{array}$ & $\begin{array}{c}\text { C22:6n-3 } \\
\text { (DHA) }\end{array}$ & $\begin{array}{c}\text { LC-PUFA } \\
n-6\end{array}$ & $\begin{array}{c}\text { LC-PUFA } \\
n-3\end{array}$ \\
\hline C18:1 t9 & 0.017 & -0.010 & $0.178^{* *}$ & $0.122^{*}$ & $-0.256^{* *}$ & $-0.124^{*}$ & $-0.265^{* *}$ & 0.024 & $-0.278^{* *}$ \\
\hline CLA c9t11 & $0.232^{* *}$ & 0.048 & $0.315^{* *}$ & $0.323^{* * *}$ & $0.125^{*}$ & $0.267^{* *}$ & $0.153^{* *}$ & $0.178^{* *}$ & $0.177^{* *}$ \\
\hline$\sum$ Trans & -0.089 & 0.038 & $0.156^{*}$ & 0.109 & -0.005 & 0.094 & -0.052 & 0.019 & -0.031 \\
\hline
\end{tabular}

AA, arachidonic acid; DGLA, dihomo-gamma-linolenic acid; DHA, docosahexaenoic acid; DPA, docosapentaenoic acid; DTA, docosatetraenoic acid; EPA, eicosapentaenoic acid; LC-PUFA, long-chain polyunsaturated fatty acid.

Pearson correlation coefficient between the respective fatty acid in maternal plasma phospholipids $\left({ }^{*} P<0.05 ;{ }^{* *} P<0.01\right)$.

Table 5. Association between the concentration of specific TFAs in maternal plasma phospholipids and atopic eczema of the infant

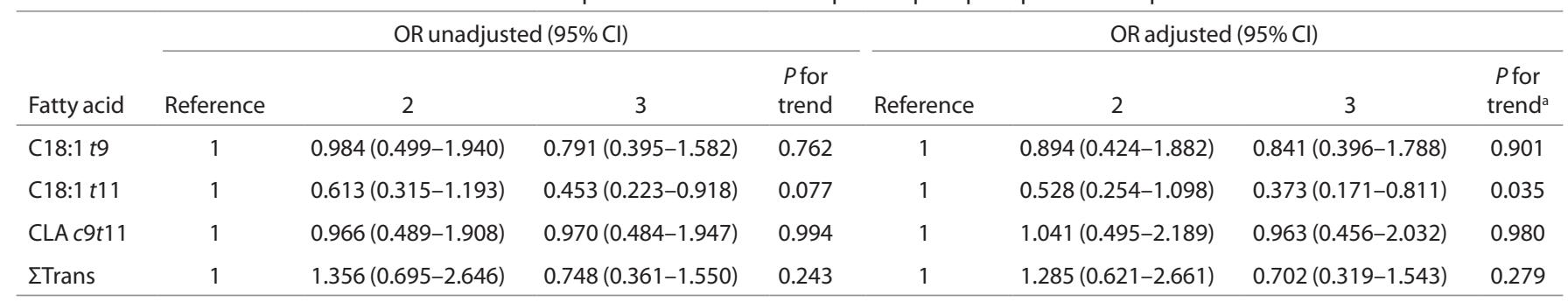

$\mathrm{Cl}$, confidence interval; OR, odds ratio; TFA, trans fatty acid.

all values were adjusted for maternal allergy, maternal atopic manifestations, maternal education, maternal smoking, sex of child, and predominant breastfeeding. The first tertile was set as the reference group.

Table 6. Association between the concentration of specific TFAs in maternal plasma phospholipids and wheeze of the infant

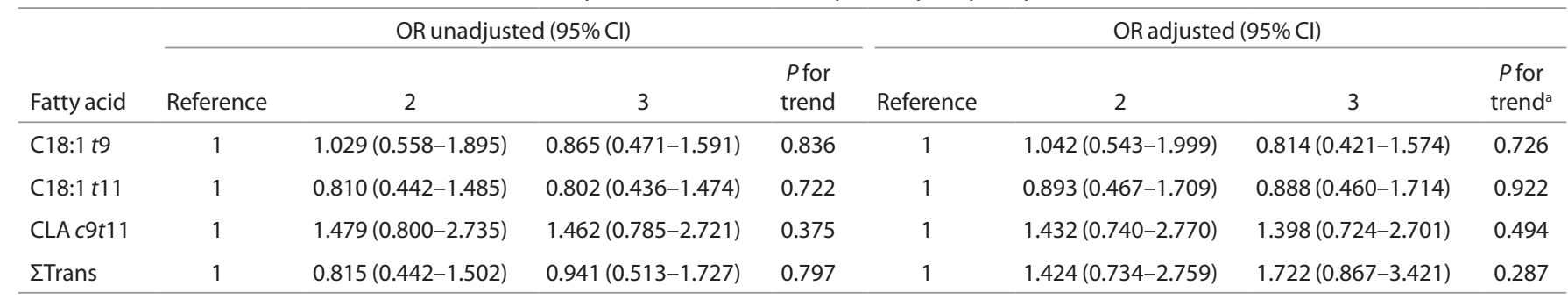

$\mathrm{Cl}$, confidence interval; OR, odds ratio; TFA, trans fatty acid.

aAll values were adjusted for maternal allergy, maternal atopic manifestations, maternal education, maternal smoking, sex of child, and predominant breastfeeding. The first tertile was set as the reference group.

pregnancy by means of FFQs. The FFQ used in this study was validated and is suitable for the assessment of dietary intake (22). The dietary intakes of FAs in this cohort were similar to those observed in several other populations in Belgium, Canada, the United States, and the United Kingdom (23-26). In our study, the consumption of LA $(11.54 \mathrm{~g} / \mathrm{d})$ and ALA $(1.10 \mathrm{~g} / \mathrm{d})$ was higher than recommended for pregnant women (27). However, the mean DHA intake in early pregnancy did not differ from the current recommendation of 0.20 to $0.30 \mathrm{~g} / \mathrm{d}$ for pregnant women $(27,28)$. The average consumption of total TFAs in pregnant women was $1.32 \mathrm{~g} / \mathrm{d}$. This value is lower than that reported in other groups of the Spanish population $(2.1 \mathrm{~g} / \mathrm{d})(29)$.

In general, the fatty acid profile of plasma phospholipids in our study was similar to those observed in other populations of pregnant women $(23,30)$. Dietary intake of the most important n-3 LC-PUFAs, such as EPA and DHA, directly correlated with respective FA concentrations in maternal plasma phospholipids. These results are consistent with those reported by other authors and confirm that n-3 LC-PUFA concentrations in plasma depend more on maternal dietary intake than on endogenous conversion (26,31). However, no correlation between $\mathrm{AA}$ intake and $\mathrm{AA}$ in plasma phospholipids was found. This observation could be attributed to the endogenous conversion of LA to AA and the high AA nutritional requirements of the fetus.

The total TFA concentrations in maternal lipids $(0.80 \%$ of total FAs) are in concordance with the amounts reported in several other studies (range from 0.50 to $2.5 \%)(21,30)$. We found that the correlation between the FFQ estimates of total TFAs and the total TFA content of maternal plasma was close to zero. The weak correlation may reflect differences in the metabolic pathways of TFAs compared with those of n-3 or n-6 LC-PUFAs. A possible explanation for this finding is that TFA deposition in tissues is selective, thus adipose tissue and liver generally contain higher concentrations of these FAs than 


\section{Articles | Chisaguanoet al.}

blood or other tissues (32). On the other hand, negative correlation between maternal intake of total TFA and rumenic acid concentration in maternal plasma indicates rumenic acid metabolized better than other TFA isomers and may be required more amount during first trimester of fetus development compared to vaccenic acid. The present results are relevant because they provide additional information about the relationships between dietary and plasma measures of TFA status among a relatively large population of women with a modest intake of TFAs.

In this population-based birth cohort study, we analyzed the FA composition of plasma in pregnant women, focusing on specific trans C18:1 isomers (elaidic and vaccenic acid), rumenic acid (c9t11-CLA), and total TFAs. To elucidate the potentially different relations of specific TFA isomers with atopic outcomes, the major dietary sources of each isomer in the population need to be explained. To our knowledge, elaidic acid is a marker of TFAs of industrial origin (partial hydrogenation of vegetable oils), while vaccenic acid and rumenic acid are the major markers of ruminant fat $(11,33)$. It has been proposed that TFA isomers (e.g., from oleic acid and LA) inhibit the enzymes involved in n- 6 and n-3 LC-PUFA synthesis $(\Delta 5$ and $\Delta 6$-desaturase). Thus, these isomers appear to impair the desaturation of LA and ALA to AA and DHA, respectively. These LC-PUFAs play central roles in infant growth, neural development, and immune function (eicosanoid metabolism) $(34,35)$. Therefore, TFAs may produce intrauterine changes during the normal fetal development.

Many studies have reported negative associations between total TFAs and n- 6 and n-3 LC-PUFA concentrations in various compartments (e.g., plasma and erythrocytes) $(8,21,36)$. However, no data are available on the relationships between industrial and ruminant TFAs (as separate groups) and LC-PUFAs. In a small study population (55 mother-child pairs), Enke et al. (37) found a negative correlation between elaidic acid and n-3 LC-PUFA (total, DPA, and DHA) in fetal but not in maternal plasma, while no correlations between vaccenic acid and LC-PUFAs in either maternal or fetal plasma were found. Our data are in disagreement with those findings as we observed that elaidic acid correlated negatively and vaccenic acid positively with n-6 (total and AA) and n-3 LC-PUFA (total, EPA, and DHA) concentrations in the plasma phospholipids of pregnant women; in addition, we detected a direct correlation between rumenic acid and n-3 LC-PUFA in maternal plasma, thereby confirming the various adverse and beneficial effects of industrial and ruminant TFAs on plasmatic LC-PUFA concentrations. Thus, rumenic acid could have protective effect since this FA is positively correlated with n-3 LC-PUFA, which has an anti-inflammatory mode of action. The differences between our findings and those of Enke et al. could be attributed to numerous factors, including the sample size, the method of analysis applied, as well as the plasma fractions examined (phospholipids vs. total plasma).

Our results also show that higher maternal vaccenic acid concentrations at 12 wk of gestation were associated with a decreased risk of atopic eczema in 1-y-old infants. The anti-inflammatory properties of vaccenic acid have not been extensively investigated. Thus, only one recent study, using animal models, has demonstrated the protective effect of high vaccenic and rumenic acid on allergic dermatitis. These FAs independently and/or jointly inhibit several facets of dermatitis (including skin leukocytosis and eosinophilia) and allergen-specific immunoglobulin E production (19). In a Dutch cohort of mother-infant pairs, Thijs et al. (38) found that high concentrations of vaccenic and rumenic acids in human breast milk were associated with a lower risk of atopic eczema and allergic sensitization in children. Accordingly, ours is the first study in a Spanish cohort to analyze prenatal exposure to vaccenic acid and the risk of atopic eczema in infants. The mother's intake of vaccenic acid during pregnancy and lactation may protect against the development of atopic eczema in early infancy. In contrast, no associations were confirmed between maternal elaidic and rumenic levels and the development of atopic eczema in 1-y-old infants. Further research involving a larger sample is required to confirm our findings.

With regard to wheeze, we did not find an association with prenatal exposure to elaidic, vaccenic, or rumenic acid concentrations through maternal plasma. In this context, the wheeze phenotype at 1-y-old is complex. Only in a minority of infants are early wheezing episodes related to a predisposition to asthma, and many infants have episodes of wheezing that are linked to viral respiratory illness $(17,39)$. These considerations may explain why we found no associations. Chatzi et al. (17) suggested that a high intake of dairy products, especially milk, during pregnancy was significantly associated with a decreased risk of wheeze in the first year of life. However, we did not find evidence of associations between ruminant TFAs and risk of wheeze. Further research involving a larger sample is needed in this field.

Our study has some limitations. We acknowledge the relatively small sample size used and recognize that a large-scale multicenter trial would be appropriate. However, it is important to note that this subsample is representative of general population with regard to FAs levels. Although comprehensive data on diet, lifestyle, and other risk factors allowed us to adjusted our statistical models for potential confounders, we cannot rule out residual confounding. This lack of data may influence relationships of interest.

To our knowledge, no previous studies have evaluated the contribution of early exposure to specific TFAs of distinct origin to the development of atopic diseases. Our findings suggest that high maternal concentrations of vaccenic acid during first trimester of pregnancy may protect against the development of atopic eczema in 1-y-old infants. Further analysis will be carried out to assess relationships of maternal TFA exposure with atopic outcomes in early childhood.

\section{METHODS}

\section{Study Population and Design}

Data for this study were obtained from a population-based pregnancy cohort set up in Sabadell (Spain) as part of the INMA-INfancia y Medio Ambiente Project (40). This study was approved by the Clinical Research Ethics Committee of the Municipal Institute of Health Care 
and the Hospital of Sabadell (Spain). Written informed consent was obtained from all the women who participated in the cohort study.

A detailed description of the Sabadell cohort has been provided elsewhere $(41,42)$. A total of 657 pregnant women were enrolled between July 2004 and July 2006 during their visits to the public health center in the first trimester of pregnancy. In this study, we included only women who had completed a FFQ in the first trimester and those for whom their babies' data on atopic outcomes were available $(75.5 \%$; $n=496$ ). During pregnancy and the first year postpartum, information on maternal characteristics and atopic outcome were obtained through questionnaires administered to the mothers at various time points (12 and $32 \mathrm{wk}$ of gestation and at 6 and 14 mo postpartum).

Maternal blood samples were extracted at $12 \mathrm{wk}$ of gestation. Plasmatic phospholipid FAs of a subset of 274 samples were analyzed for this study.

\section{Dietary Assessment During Pregnancy}

A detailed description of the dietary assessment has been published elsewhere (17). In the INMA-Sabadell cohort, a FFQ was administered by trained interviewers to assess common food and nutrient intake during the first trimester of pregnancy. That FFQ was previously validated in a group of pregnant women of the INMA Project in Valencia and included 100 food items (22). The response to each food item was converted into the average consumption in grams per day. Thus, nutrient intake was calculated by multiplying the frequency of consumption of each food item by the nutrient composition of the portion size specified in the FFQ and by summing across all foods to obtain a total nutrient intake per each participant. We used the residual method to estimate calorie-adjusted values for nutrient intake (43).

\section{Atopic Manifestations Definition}

Maternal atopy was defined as a positive response (levels $\geq 0.35 \mathrm{KU} / \mathrm{l}$ ) to any of the following three allergens: cat dander, dust mite, and mixed grass pollen (Poaceae plants), which are the most common allergens in Spain. These criteria were used in previous studies $(7,42)$.

Information about the outcomes of interest (atopic eczema and wheezing) was obtained from parents through two questionnaires completed when the child was 6 and 14 mo old. Wheezing was defined as a positive answer to the question "Has ever your child experienced whistling or wheezing from the chest, but not noisy breathing from the nose since birth to six months, and from 7 to 14 months?".

Atopic eczema was defined as a positive response to the question "Has your child ever experienced atopic eczema since birth to six months, and from 7 to 14 months?". Thus, children whose parents reported that they had been diagnosed with eczema during both periods (recurrent eczema) were classified as atopic cases.

\section{Fatty Acid Composition of Plasma Phospholipids in Mothers}

The blood samples from the pregnant women were centrifuged, and plasma samples were stored at $-80{ }^{\circ} \mathrm{C}$ until analysis. The FA composition of plasma phospholipids was determined using the method developed in our laboratory and described elsewhere (44). Plasma lipids were extracted using dichloromethane:methanol (2:1), and phospholipids were separated by solid-phase extraction $(100 \mathrm{mg}$ aminopropyl, 96-well plates). Phospholipids were converted to FA methyl esters by heating with sodium methylate in methanol $0.5 \mathrm{~mol} / \mathrm{l}$ and boron trifluoride methanol solution $(14 \% \mathrm{v} / \mathrm{v})$. FA methyl esters were separated and quantified by fast gas chromatography with flame ionization detection. The identities of FA methyl ester peaks were determined by comparison of their relative retention times with those of standard FA methyl ester mix solutions (Sigma Aldrich, St Louis, MO). We focused our attention on n-3 and n-6 PUFAs and individual TFAs from C18:1 and C18:2. The results were expressed in relative amounts (\% of total FAs).

\section{Statistical Analysis}

Means and SDs were used to describe continuous variables. The Kolmogorov-Smirnov test was used to study the normal distribution of data, and nonnormally distributed data were naturally log transformed. Correlations between FA intake and maternal FA status, namely, TFAs and LC-PUFAs, were determined using Pearson's rank correlation coefficient. The FAs of interest were classified into three categories on the basis of the tertile distribution within the total cohort. Multivariable regression models were used to examine the association of specific TFAs in maternal plasma (using the first tertile as a reference) and atopic outcomes in infants after adjusting for potential confounders. These confounders included characteristics that have an established or potential associations with eczema and wheezing in the first year of life and include the following: maternal age; maternal educational level (primary schooling or lower, secondary schooling, and higher education); maternal smoking during pregnancy (yes/no); maternal history of atopic manifestations (yes/ no); maternal allergy (yes/no); infant gender (male or female); and duration of predominant breastfeeding (weeks). All statistical analyses were performed on SPSS 20.0 for Windows (SPSS, Chicago, IL).

\section{ACKNOWLEDGMENTS}

We thank Silvia Fochs, Anna Sánchez, and Nuria Pey for their assistance in contacting the families and administering the questionnaires. Special thanks go to the participants in the INMA study for their generous donations. We also thank Toffa Evans for his help with English grammar and expression.

\section{STATEMENT OF FINANCIAL SUPPORT}

We thank the Spanish Ministry of Economy and Competitiveness for the predoctoral grant awarded to A.M.C. and financial support for the research project AGL2009-09730/ALI. We also thank the Spanish Ministry of Economy and Competitiveness for a postdoctoral Juan de la Cierva fellowship awarded to R.M.

Disclosure: The authors declare no conflict of interest.

\section{REFERENCES}

1. Asher MI, Montefort S, Björkstén B, et al.; ISAAC Phase Three Study Group. Worldwide time trends in the prevalence of symptoms of asthma, allergic rhinoconjunctivitis, and eczema in childhood: ISAAC Phases One and Three repeat multicountry cross-sectional surveys. Lancet 2006;368:733-43.

2. Notenboom ML, Mommers M, Jansen EH, Penders J, Thijs C. Maternal fatty acid status in pregnancy and childhood atopic manifestations: KOALA Birth Cohort Study. Clin Exp Allergy 2011;41:407-16.

3. Nwaru BI, Erkkola M, Lumia M, et al. Maternal intake of fatty acids during pregnancy and allergies in the offspring. Br J Nutr 2012;108:720-32.

4. Dunder T, Kuikka L, Turtinen J, Räsänen L, Uhari M. Diet, serum fatty acids, and atopic diseases in childhood. Allergy 2001;56:425-8.

5. Miyake Y, Okubo H, Sasaki S, Tanaka K, Hirota Y. Maternal dietary patterns during pregnancy and risk of wheeze and eczema in Japanese infants aged 16-24 months: the Osaka Maternal and Child Health Study. Pediatr Allergy Immunol 2011;22:734-41.

6. Sausenthaler S, Koletzko S, Schaaf B, et al.; LISA Study Group. Maternal diet during pregnancy in relation to eczema and allergic sensitization in the offspring at $2 y$ of age. Am J Clin Nutr 2007;85:530-7.

7. Montes R, Chisaguano AM, Castellote AI, Morales E, Sunyer J, LópezSabater MC. Fatty-acid composition of maternal and umbilical cord plasma and early childhood atopic eczema in a Spanish cohort. Eur J Clin Nutr 2013;67:658-63.

8. Decsi T, Koletzko B. Do trans fatty acids impair linoleic acid metabolism in children? Ann Nutr Metab 1995;39:36-41.

9. Innis SM. Trans fatty intakes during pregnancy, infancy and early childhood. Atheroscler Suppl 2006;7:17-20.

10. Larqué E, Pérez-Llamas F, Puerta V, et al. Dietary trans fatty acids affect docosahexaenoic acid concentrations in plasma and liver but not brain of pregnant and fetal rats. Pediatr Res 2000;47:278-83.

11. Albuquerque TG, Costa HS, Castilho MC, Sanches-Silva A. Trends in the analytical methods for the determination of trans fatty acids content in foods. Trends Food Sci Tech 2011;22:543-60.

12. Motard-Bélanger A, Charest A, Grenier G, et al. Study of the effect of trans fatty acids from ruminants on blood lipids and other risk factors for cardiovascular disease. Am J Clin Nutr 2008;87:593-9.

13. Wang Y, Jacome-Sosa MM, Vine DF, Proctor SD. Beneficial effects of vaccenic acid on postprandial lipid metabolism and dyslipidemia: impact of natural trans-fats to improve CVD risk. Lipid Tech 2010;22:103-6. 
14. Jiang J, Wolk A, Vessby B. Relation between the intake of milk fat and the occurrence of conjugated linoleic acid in human adipose tissue. Am J Clin Nutr 1999;70:21-7.

15. Kummeling I, Thijs $\mathrm{C}$, Huber $\mathrm{M}$, et al. Consumption of organic foods and risk of atopic disease during the first 2 years of life in the Netherlands. Br J Nutr 2008;99:598-605.

16. Maslova E, Halldorsson TI, Strøm M, Olsen SF. Low-fat yoghurt intake in pregnancy associated with increased child asthma and allergic rhinitis risk: a prospective cohort study. J Nutr Sci 2012;1:e5.

17. Chatzi L, Garcia R, Roumeliotaki T, et al.; INMA study group; RHEA study group. Mediterranean diet adherence during pregnancy and risk of wheeze and eczema in the first year of life: INMA (Spain) and RHEA (Greece) mother-child cohort studies. Br J Nutr 2013;110:2058-68.

18. Calvani M, Alessandri C, Sopo SM, et al.; Lazio Association of Pediatric Allergology (APAL) Study Group. Consumption of fish, butter and margarine during pregnancy and development of allergic sensitizations in the offspring: role of maternal atopy. Pediatr Allergy Immunol 2006;17:94102.

19. Sun X, Zhang J, Macgibbon AK, Black P, Krissansen GW. Bovine milk fat enriched in conjugated linoleic and vaccenic acids attenuates allergic dermatitis in mice. Clin Exp Allergy 2011;41:729-38.

20. Kanwar RK, Macgibbon AK, Black PN, et al. Bovine milk fat enriched in conjugated linoleic and vaccenic acids attenuates allergic airway disease in mice. Clin Exp Allergy 2008;38:208-18.

21. Decsi T, Boehm G. trans Isomeric fatty acids are inversely related to the availability of long-chain PUFAs in the perinatal period. Am J Clin Nutr 2013;98:543S-8S.

22. Vioque J, Navarrete-Muñoz EM, Gimenez-Monzó D, et al.; INMAValencia Cohort Study. Reproducibility and validity of a food frequency questionnaire among pregnant women in a Mediterranean area. Nutr J 2013;12:26.

23. De Vriese SR, Matthys C, De Henauw S, De Backer G, Dhont M, Christophe AB. Maternal and umbilical fatty acid status in relation to maternal diet. Prostaglandins Leukot Essent Fatty Acids 2002;67:389-96.

24. Innis SM, Elias SL. Intakes of essential n- 6 and n-3 polyunsaturated fatty acids among pregnant Canadian women. Am J Clin Nutr 2003;77:473-8.

25. Thomas B, Ghebremeskel K, Lowy C, Crawford M, Offley-Shore B. Nutrient intake of women with and without gestational diabetes with a specific focus on fatty acids. Nutrition 2006;22:230-6.

26. Donahue SM, Rifas-Shiman SL, Olsen SF, Gold DR, Gillman MW, Oken E. Associations of maternal prenatal dietary intake of n-3 and n-6 fatty acids with maternal and umbilical cord blood levels. Prostaglandins Leukot Essent Fatty Acids 2009;80:289-96.

27. Simopoulos AP, Leaf A, Salem N Jr. Essentiality of and recommended dietary intakes for omega- 6 and omega-3 fatty acids. Ann Nutr Metab 1999;43:127-30.

28. Koletzko B, Lien E, Agostoni C, et al.; World Association of Perinatal Medicine Dietary Guidelines Working Group. The roles of long-chain polyunsaturated fatty acids in pregnancy, lactation and infancy: review of current knowledge and consensus recommendations. J Perinat Med 2008; 36:5-14.
29. Craig-Schmidt MC. World-wide consumption of trans fatty acids. Atheroscler Suppl 2006;7:1-4.

30. Vlaardingerbroek H, Hornstra G. Essential fatty acids in erythrocyte phospholipids during pregnancy and at delivery in mothers and their neonates: comparison with plasma phospholipids. Prostaglandins Leukot Essent Fatty Acids 2004;71:363-74.

31. Tu WC, Cook-Johnson RJ, James MJ, Mühlhäusler BS, Gibson RA. Omega-3 long chain fatty acid synthesis is regulated more by substrate levels than gene expression. Prostaglandins Leukot Essent Fatty Acids 2010;83:61-8

32. Garland M, Sacks FM, Colditz GA, et al. The relation between dietary intake and adipose tissue composition of selected fatty acids in US women. Am J Clin Nutr 1998;67:25-30.

33. Seppänen-Laakso T, Laakso I, Backlund P, Vanhanen H, Viikari J. Elaidic and trans-vaccenic acids in plasma phospholipids as indicators of dietary intake of 18:1 trans-fatty acids. J Chromatogr B Biomed Appl 1996;687:371-8.

34. Dirix CE, Kester AD, Hornstra G. Associations between neonatal birth dimensions and maternal essential and trans fatty acid contents during pregnancy and at delivery. Br J Nutr 2009;101:399-407.

35. Duttaroy AK. Transport of fatty acids across the human placenta: a review. Prog Lipid Res 2009;48:52-61.

36. Elias SL, Innis SM. Infant plasma trans, $n-6$, and n-3 fatty acids and conjugated linoleic acids are related to maternal plasma fatty acids, length of gestation, and birth weight and length. Am J Clin Nutr 2001;73:807-14.

37. Enke U, Jaudszus A, Schleussner E, Seyfarth L, Jahreis G, Kuhnt K. Fatty acid distribution of cord and maternal blood in human pregnancy: special focus on individual trans fatty acids and conjugated linoleic acids. Lipids Health Dis 2011;10:247.

38. Thijs C, Müller A, Rist L, et al. Fatty acids in breast milk and development of atopic eczema and allergic sensitisation in infancy. Allergy 2011;66:5867.

39. Martinez FD, Wright AL, Taussig LM, Holberg CJ, Halonen M, Morgan WJ. Asthma and wheezing in the first six years of life. The Group Health Medical Associates. N Engl J Med 1995;332:133-8.

40. Guxens M, Ballester F, Espada M, et al.; INMA Project. Cohort Profile: the INMA-INfancia y Medio Ambiente-(Environment and Childhood) Project. Int J Epidemiol 2012;41:930-40.

41. Morales E, Bustamante M, Gonzalez JR, et al. Genetic variants of the FADS gene cluster and ELOVL gene family, colostrums LC-PUFA levels, breastfeeding, and child cognition. PLoS One 2011;6:e17181.

42. Morales E, García-Esteban R, Guxens M, et al. Effects of prolonged breastfeeding and colostrum fatty acids on allergic manifestations and infections in infancy. Clin Exp Allergy 2012;42:918-28.

43. Willett WC. Reproducibility and validity of food-frequency questionnaires. In: Walter Willett C, eds. Nutritional Epidemiology. New York: Oxford University Press, 1998:101-47.

44. Chisaguano AM, Lozano B, Molto-Puigmarti C, Castellote AI, Rafecas M, Lopez-Sabater MC. Elaidic acid, vaccenic acid and rumenic acid (c9,t11CLA) determination in human plasma phospholipids and human milk by fast gas chromatography. Anal Methods 2013;5:1264-72. 